\title{
Evaluation of Different Phenotypic Methods Versus Polymerase Chain Reaction for Detection of Plasmid Mediated AmpC $\beta$-Lactamase-Producing Strains of Proteus mirabilis
}

\author{
Rania A. Amar ${ }^{1}$ and Karim A. Montasser ${ }^{2 *}$ \\ ${ }^{1}$ Department of Clinical and Chemical Pathology, Faculty of Medicine, \\ Ain Shams University, Cairo, Egypt \\ ${ }^{2}$ Department of Clinical and Chemical Pathology, Faculty of Medicine, \\ Helwan University, Cairo, Egypt \\ *Corresponding author
}

\section{A B S T R A C T}

\section{Keywords}

Plasmid mediated AmpC $\beta$ lactamases,

Proteus Mirabilis, Cephamycin-Hodge test, Tris-EDTA disk test, Combination disk test, PCR.

\section{Article Info}

\section{Accepted:}

28 September 2017

Available Online:

10 November 2017
In recent years, the prevalence of infections with multidrug resistant Enterobacteriaceae has steadily increased. Enterobacteriaceae producing AmpC $\beta$-lactamases (AmpCs) have become a major therapeutic challenge. The detection of AmpC-producing Proteus mirabilis is of significant clinical relevance, as this may lead to inappropriate antimicrobial regimens and therapeutic failure. The aim of this study is to evaluate and comparing routinely phenotypic methods in detection of resistance with molecular methods. From this study, it can be concluded that cephamycin-Hodge test is the most sensitive, specific, interpretable and efficient test for detection of AmpC $\beta$-lactamases in clinical isolates of Proteus mirabilis, compared to the molecular method.

\section{Introduction}

$\beta$-lactam antibiotics account for approximately $50 \%$ of global antibiotic consumption which has considerably increased the resistance in Gram negative bacteria. Amp C $\beta$-lactamase production is one of the commonest causes of resistance to $\beta$ lactam antibiotics among Gram negative bacteria (1).

Proteus mirabilis, is a major organism among normal flora and it causes a wide variety of intestinal and extra- intestinal diseases, such as bacteremia, pneumonia, and other infections as wound, chest and even meningitis $(2,3)$. As a result of antibiotics abuse, the problem of having different antibiotic resistant patterns among microorganisms had extensively emerged.

The main cause of the emergence of such problem is being away from applying measures and guidelines of infection control regarding programs of antibiotics stewardship in hospitals. This had led to increase the 
magnitude of the problem and also the spread of this problem worldwide (4).

Different methods of AmpC $\beta$-lactamases group $\mathrm{C}$ detection have been described. Screening tools include resistance to cephamycins and/or ceftazidime (5), retaining cefepime susceptibility (6), modified cefoxitin Hodge test (7) and Tris-EDTA disc test(8), inhibitor-based assays (e.g., using boronic acid compounds (9) or cloxacillin,(10) and rapid chromogenic assays (11). Those methods are not used for routine work in clinical microbiology laboratories and for the diagnosis of different AmpC $\beta$-lactamases (12).

There is a high need for simple methods to observe the resistance of plasmid AmpC $\beta$ lactamase. The aim of this study was to evaluate efficacy of different phenotypic methods compared to PCR as a gold standard test for rapid and accurate detection of AmpC $\beta$-lactamases.

\section{Materials and Methods}

This study was conducted on fifty clinical isolates of Proteus spp. isolated from different clinical specimens referred to Microbiology Central Laboratory of Helwan University Hospitals in the period from May to December 2015. Specimens studied were 21 pus specimens, 10 urine specimens, 9 wound swabs, 5 sputum specimens, 3 blood specimens, 1 endotracheal tube specimen and 1 stool specimen. All samples were collected under aseptic conditions, and isolates of proteus species were stored in aliquots on trypton soya broth (Oxoid, UK.) at $-70^{\circ} \mathrm{C}$ till used.

Isolates were directly sub-cultured on blood and MacConkey agar plates using a sterile bacteriological loop. Incubation of plates was done at $37^{\circ} \mathrm{C}$ in aerobic condition. Plates were examined after overnight incubation for separate colonies. Isolates were identified by gram stain, culture characters and biochemical reactions. Antibiotic susceptibility testing was performed using susceptibility test disks (Becton Dickinson, Germany), and CLSI guidelines.

Susceptibility testing was performed on Muller- Hinton agar (bioMerieux, France), using overnight cultures at a $0.5 \mathrm{McFarland}$ standard followed by incubation at $35 \mathrm{C}$ for 16-18h.

\section{Detection of AmpC B- lactamases}

Phenotypic detection of AmpC $\beta$ Lactamase

\section{Cephamycin Hodge test}

Cephamycin Hodge test using cefoxitin disk $30 \mu \mathrm{g}$ and E.coli reference strain ATCC 25922 (supplied by NAMRU-3) was done and interpreted according to Nassim et al., (13).

\section{Tris-EDTA (TE)-disk test}

A suspension of the cefoxitin susceptible strain of E.coli ATCC 25922, and results were interpreted according to Singhal et al., (14).

\section{Combination-disk test with boronic acid}

Disks containing cefoxitin $30 \mu \mathrm{g}$ and cefoxitin plus $400 \mu \mathrm{g}$ of boronic acid were used and the test was done according to Song et al., (15).

\section{Molecular detection of AmpC $\beta$ - Lactamases}

\section{Preparation of template DNA}

Cells were harvested in a microcentrifuge tube by centrifugation for $10 \mathrm{~min}$ at $5000 \mathrm{xg}$ 
(7500rpm). The supernatant was discarded. The DNA Mini spin column was placed in a new $2 \mathrm{ml}$ collection tube, with added 500ul buffer in 2 steps successively using AW1 then AW2. Finally the DNA Mini spin column was placed in a clean $1.5 \mathrm{ml}$ microcentrifuge tube and 100ul buffer AE was pipette directly onto the DNA membrane centrifugation for $1 \mathrm{~min}$ (8000rpm) to elute.

\section{Protocol for Real Time PCR}

Real time PCR was performed for amplification of FOX-1geneusing the method described by Perez-Perez and Hanson (16). PCR was performed in a DNA thermal cycler (Biometra, Germany) with a final volume of 50ulin a $0.5-\mathrm{ml}$ thin- walled tubes. For the detection of FOX-1 gene 5-AAC ATG GGG TAT CAG GGA GAT G-3 was used as a forward primer (corresponding to nucleotides 1475-1496) and 5-CAA AGC GCG TAA CCG GAT TGG-3 was used as a reverse primer (corresponding to nucleotides 16641644) expected amplicon size 190bp.The template DNA ( $\leq 500 \mathrm{ng} /$ reaction) was added to the individual PCR tubes containing the master mix. The thermal cycler was programmed according to Alper et al., (17).

\section{Data analysis}

Performances of various phenotypic tests in the detection of AmpC $\beta$-Lactamases were evaluated to their PCR results.

\section{Interpretation}

The greenish horizontal line in the graph of Figure 1 is the threshold line at which the fluorescence begins to be detected (The point at which the amplification plot crosses the threshold is the cycle threshold $=\mathrm{Ct}$ ). The Tm of samples which were identical or close to that of positive control was considered the gene of target as shown in Figure 2.

\section{Results and Discussion}

In our study, out of 50 specimens, 21(42.0\%) were negative by both cephamycin-Hodge test and PCR and 29(58.0\%) out of 50 specimens were positive by PCR, 22(75.9\%) of which were positive by both tests while 7 (24.1\%) specimens were negative by cephamycinHodge test and positive by PCR. Agreement between both methods was $86.0 \%$. There was a statistical significant agreement between them $(\mathrm{P}<0.05)($ Table 1 and Figure 3$)$.

Out of 50 specimens, 21(42.0\%) were negative by both Tris-EDTA disk test and PCR and 29(58.0\%) out of 50 specimens were positive by PCR, 11(37.9\%) of which were positive by both tests while $18(62.1 \%)$ specimens were negative by Tris-EDTA disk test and positive by PCR. Agreement between both methods was $64.0 \%$. There was a statistical significant disagreement between them $(\mathrm{P}<0.05)$ (Table 2 and Figure 4). Out of 50 specimens, 21(42.0\%) were negative by both combination disk test with boronic acid and PCR and 29(58.0\%) out of 50 specimens were positive by PCR, 17(58.6\%) of which were positive by both tests while 12 (41.4\%) specimens were negative by combination disk test with boronic acid and positive by PCR. Agreement between both methods was $76.0 \%$. There was a statistical significant disagreement between them $(\mathrm{P}<0.05)$ (Table 3 and Figure 5).

27 specimens (96.4\%) were negative by both Cephamycin-Hodge test and Tris-EDTA disk test and 10 specimens $(45.5 \%)$ were positive by both tests while 12 specimens $(54.5 \%)$ were positive by Cephamycin-Hodge test and negative by Tris-EDTA disk test and 1 specimen $(3.6 \%)$ was positive by Tris-EDTA disk test and negative by Cephamycin-Hodge test. There was a statistical significant difference between them $(\mathrm{P}<0.05)$ (Table 4 and Figure 6). 
26 specimens (92.9\%) were negative by both Cephamycin-Hodge test and combinationdisk test with boronic acid and 15 specimens $(68.2 \%)$ were positive by both tests while 7 specimens (31.8\%) were positive by Cephamycin-Hodge test and negative by combination-disk test with boronic acid and 2 specimens $(7.1 \%)$ was positive by combination-disk test with boronic and negative by Cephamycin-Hodge test. There was a statistical significant difference between them $(\mathrm{P}<0.05)$ (Table 5 and Figure 7). 33 specimens $(84.6 \%)$ were negative by both Tris-EDTA disk test and combination- disk test with boronic acid and 11 specimens $(100.0 \%)$ were positive by both tests while 6 specimens $(15.4 \%)$ were negative by TrisEDTA disk test and positive by combinationdisk test with boronic acid. There was a statistical significant difference between them $(\mathrm{P}<0.05)$ (Table 6 and Figure 8).

Agreement between PCR results and phenotypic methods were $86 \%, 64 \%$ and $76 \%$ for cephamycin- Hodge test, Tris- EDTA test and combination disk test respectively with statistical significant difference between them $(\mathrm{P}<0.05)$.

Table.1 Correlation between Cephamycin-Hodge test and PCR as a reference method

\begin{tabular}{|c|c|c|c|c|c||}
\hline \multicolumn{2}{|c|}{} & \multicolumn{2}{|c|}{ PCR } & \multirow{2}{*}{ Total } \\
\cline { 4 - 6 } \multicolumn{3}{|c|}{ Cephamycin-Hodge Test } & Negative & Positive & \\
\hline \hline \multirow{3}{*}{ Negative } & Count & 21 & 7 & 28 \\
\cline { 3 - 6 } & \multirow{2}{*}{ Positive } & $\%$ & $42.0 \%$ & $24.1 \%$ & $56.0 \%$ \\
\cline { 3 - 6 } & & Count & 0 & 22 & 22 \\
\cline { 3 - 6 } & & $\%$ & $0.0 \%$ & $75.9 \%$ & $44.0 \%$ \\
\hline \multirow{2}{*}{ Total } & Count & 21 & 29 & 50 \\
\cline { 3 - 6 } & & $\%$ & $100.0 \%$ & $100.0 \%$ & $100.0 \%$ \\
\hline
\end{tabular}

Table.2 Correlation between Tris-EDTA disk test and PCR as a reference method

\begin{tabular}{|c|c|c|c|c|c|}
\hline & & & \multicolumn{2}{|c|}{ PCR } & \multirow{2}{*}{ Total } \\
\hline & & & Negative & Positive & \\
\hline \multirow{4}{*}{ Tris-EDTA disk Test } & \multirow{2}{*}{ Negative } & Count & 21 & 18 & 39 \\
\hline & & $\%$ & $42.0 \%$ & $62.1 \%$ & $78.0 \%$ \\
\hline & \multirow{2}{*}{ Positive } & Count & 0 & 11 & 11 \\
\hline & & $\%$ & $0.0 \%$ & $37.9 \%$ & $22.0 \%$ \\
\hline \multirow{2}{*}{\multicolumn{2}{|c|}{ Total }} & Count & 21 & 29 & 50 \\
\hline & & $\%$ & $100.0 \%$ & $100.0 \%$ & $100.0 \%$ \\
\hline
\end{tabular}

Table.3 Correlation between combination- disk test with boronic acid and PCR as a reference method

\begin{tabular}{|c|c|c|c|c|c|}
\hline & & & \multicolumn{2}{|c|}{ 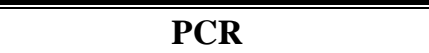 } & \multirow{2}{*}{ Total } \\
\hline & & & Negative & Positive & \\
\hline \multirow{4}{*}{$\begin{array}{c}\text { Combination disk Test with } \\
\text { BA }\end{array}$} & \multirow{2}{*}{ Negative } & Count & 21 & 12 & 33 \\
\hline & & $\%$ & $42.0 \%$ & $41.4 \%$ & $66.0 \%$ \\
\hline & \multirow{2}{*}{ positive } & Count & 0 & 17 & 17 \\
\hline & & $\%$ & $0.0 \%$ & $58.6 \%$ & $34.0 \%$ \\
\hline \multirow{2}{*}{\multicolumn{2}{|c|}{ Total }} & Count & 21 & 29 & 50 \\
\hline & & $\%$ & $100.0 \%$ & $100.0 \%$ & $100.0 \%$ \\
\hline
\end{tabular}


Table.4 Correlation between Cephamycin-Hodge test Tris-EDTA disk test

\begin{tabular}{|c|c|c|c|c|c|}
\hline & & & \multicolumn{2}{|c|}{ Cephamycin-Hodge Test } & \multirow{2}{*}{ Total } \\
\hline & & & Negative & Positive & \\
\hline \multirow{4}{*}{ Tris-EDTA disk T } & \multirow{2}{*}{ Negative } & Count & 27 & 12 & 39 \\
\hline & & $\%$ & $96.4 \%$ & $54.5 \%$ & $78.0 \%$ \\
\hline & \multirow{2}{*}{ Positive } & Count & 1 & 10 & 11 \\
\hline & & $\%$ & $3.6 \%$ & $45.5 \%$ & $22.0 \%$ \\
\hline \multirow{2}{*}{\multicolumn{2}{|c|}{ Total }} & Count & 28 & 22 & 50 \\
\hline & & $\%$ & $100.0 \%$ & $100.0 \%$ & $100.0 \%$ \\
\hline
\end{tabular}

Table.5 Correlation between cephamycin-Hodge test and combination-disk test with boronic acid

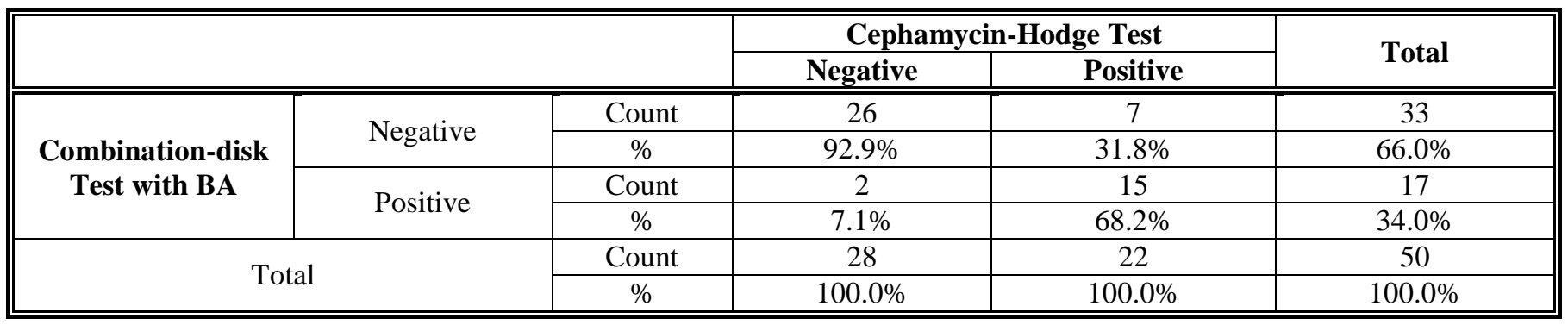

Table.6 Correlation between Tris-EDTA disk and combination- disk test with boronic acid

\begin{tabular}{|c|c|c|c|c|c|}
\hline & & & \multicolumn{2}{|c|}{ Tris-EDTA disk Test } & \multirow{2}{*}{ Total } \\
\hline & & & Negative & Positive & \\
\hline \multirow{4}{*}{$\begin{array}{c}\text { Combination disk Test } \\
\text { with BA }\end{array}$} & \multirow{2}{*}{ Negative } & Count & 33 & 0 & 33 \\
\hline & & $\%$ & $84.6 \%$ & $0.0 \%$ & $66.0 \%$ \\
\hline & \multirow{2}{*}{ Positive } & Count & 6 & 11 & 17 \\
\hline & & $\%$ & $15.4 \%$ & $100.0 \%$ & $34.0 \%$ \\
\hline \multirow{2}{*}{\multicolumn{2}{|c|}{ Total }} & Count & 39 & 11 & 50 \\
\hline & & & $100.0 \%$ & $100.0 \%$ & $100.0 \%$ \\
\hline
\end{tabular}

Fig.1 Results of syber Green real time PCR in amplification plot with cycles number on X axis and florescence on $\mathrm{Y}$ axis

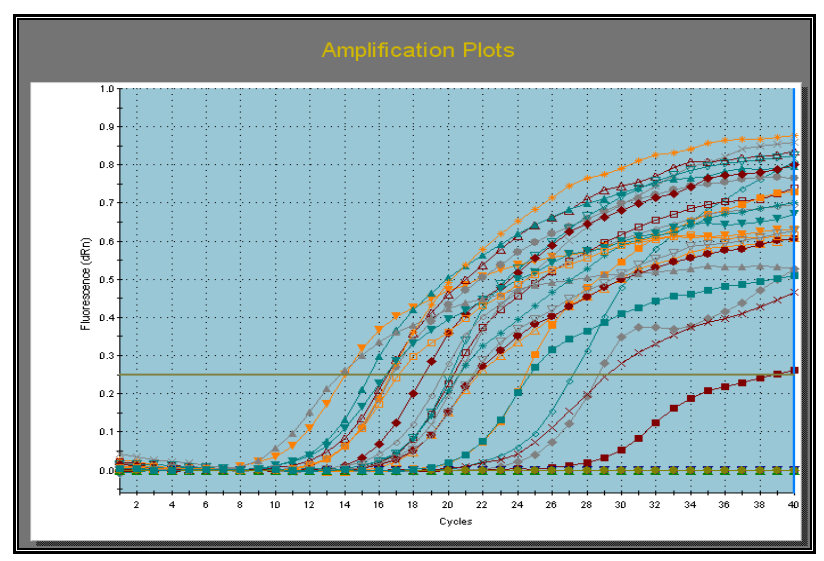


Fig.2 Results of melting curve, average $\mathrm{Tm}=77.13^{\circ} \mathrm{C}-77.72^{\circ} \mathrm{C}$

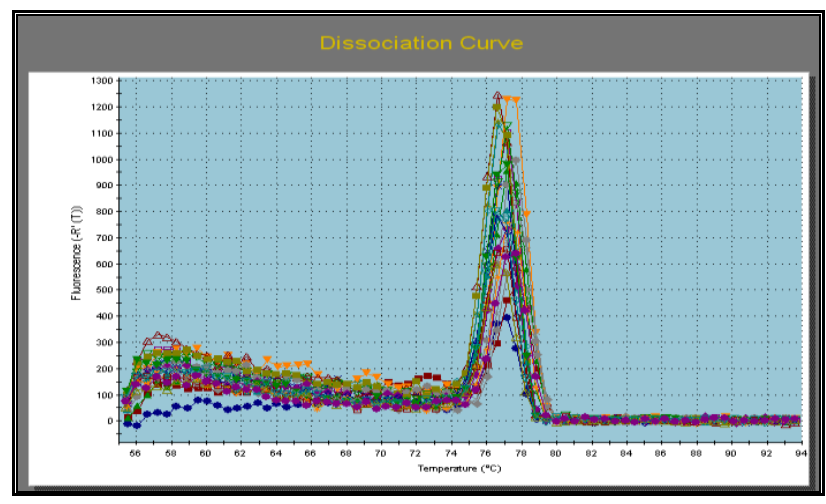

Fig.3 Evaluation of cephamycin-Hodge Test Vs PCR

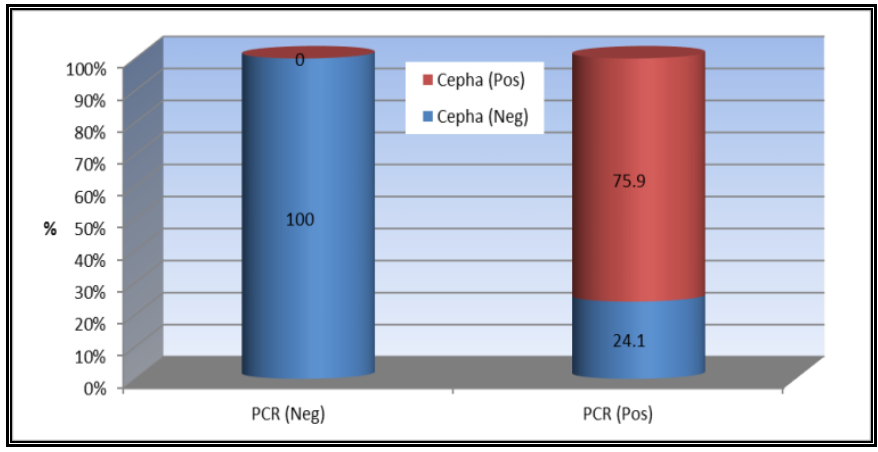

Fig.4 Evaluation of Tris-EDTA Disk Test Vs PCR

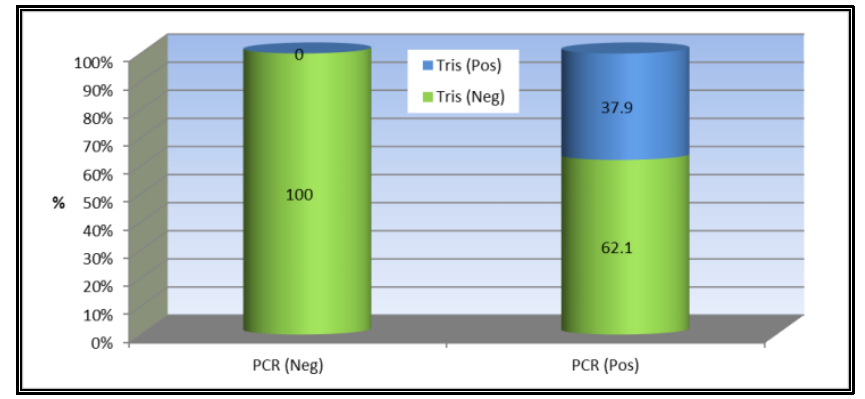

Fig.5 Evaluation of combination Disk Test with BA Vs PCR

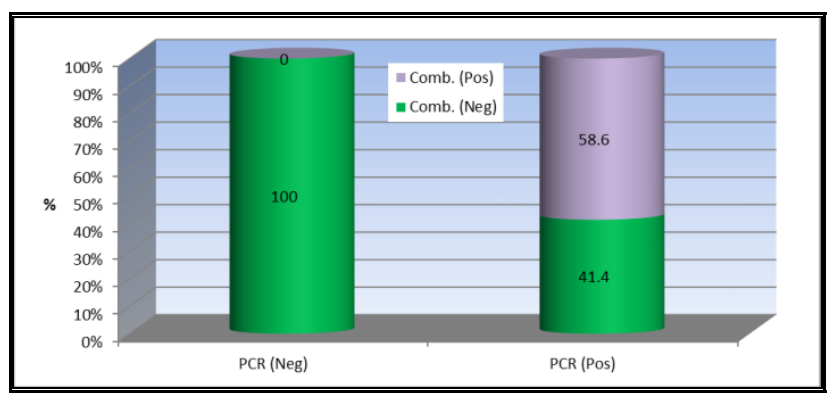


Fig.6 Correlation between cephamycin-Hodge test and Tris-EDTA disk test

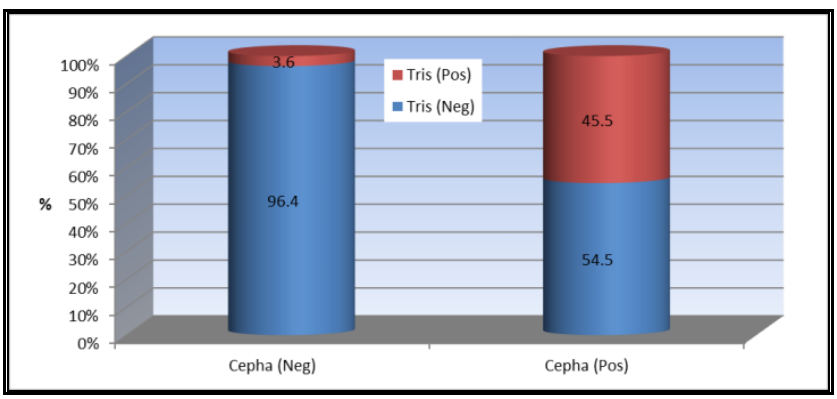

Fig.7 Correlation between cephamycin- Hodge test and combination-disk test with boronic acid

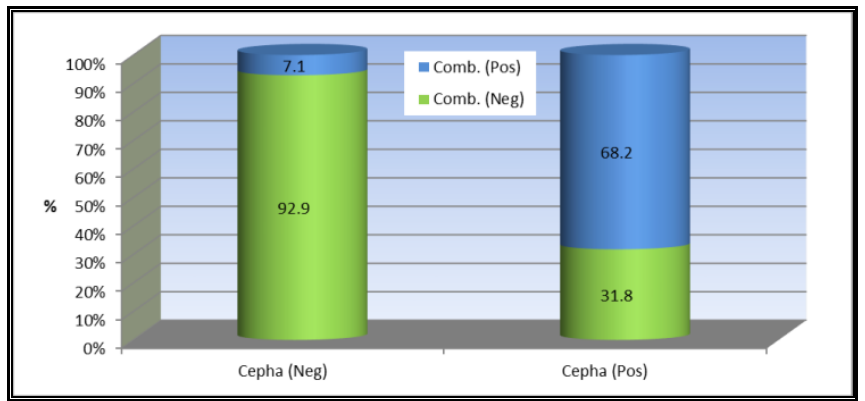

Fig.8 Correlation between Tris- EDTA disk test and Combination- disk test with boronic acid

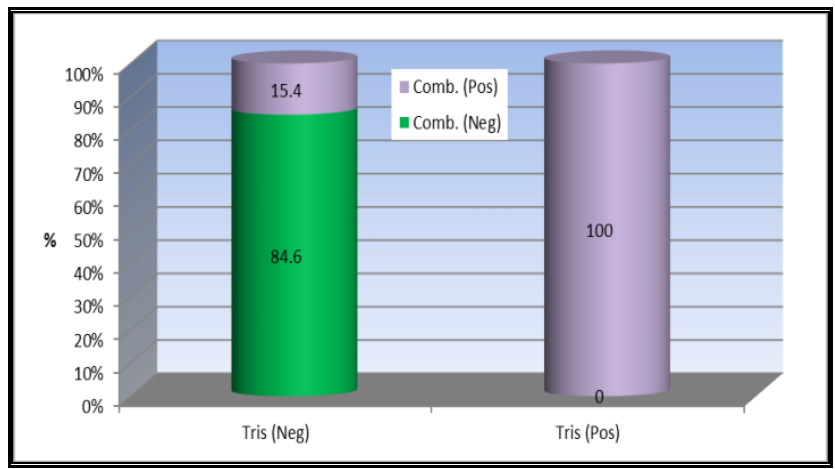

Fig.9 Comparison between all studied methods as regards evaluation criteria

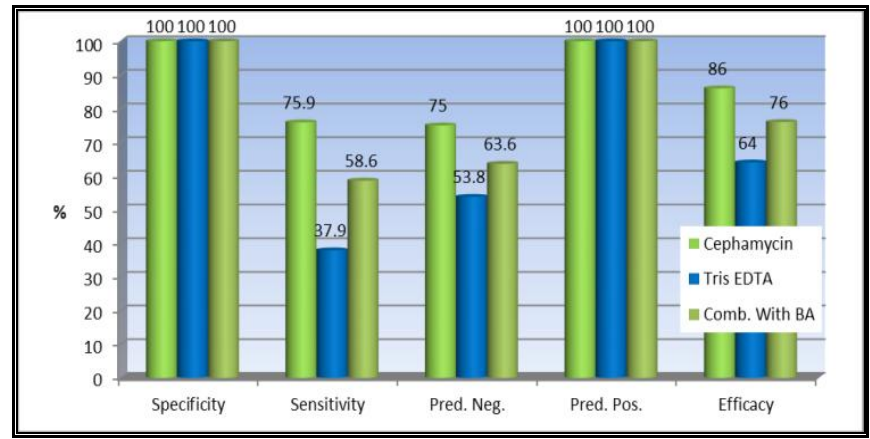


Specificity of Cephamycin-Hodge test, TrisEDTA disk test and combination-disk test with boronic acid was $(100.0 \%, 100.0 \%$ \& $100.0 \%)$ respectively, sensitivity was $(75.9 \%$, $37.9 \%$ \& $58.6 \%$ ) respectively, negative predictive value was $(75.0 \%, 53.8 \%$ \& $63.6 \%$ ) respectively, positive predictive value was $(100.0 \%, 100.0 \% \& 100.0 \%)$ respectively and the efficacy was $(86.0 \%, 64.0 \%$ \& $76.0 \%$ ) respectively (Figure 9).

In the present study, combination-disk test with $\mathrm{BA}$ detected $\mathrm{AmpC}$ producing strains in 17(58.6\%) out of 29 PCR-positive isolates. In contrast to our results, Coudron 2005 (18) screened 271 clinical isolates for cefoxitin susceptibility by the standard disk diffusion method (128 Klebsiella spp., 115 E. coli and 28 P. mirabilis isolates). Screen-positive isolates were tested for the presence of the AmpC $\beta$-lactamase by a three-dimensional method, boronic acid disk test and PCR. He found that 55 out of 271 isolates were AmpCPCR-positive, and the boronic acid disk test detected 54 of the isolates (13 Klebsiella, 38 E. coli and 3 P. mirabilis). For this reason, he concluded that the boronic acid disk test is the recommended method as sensitivity was $90 \%$ and specificity was $98.2 \%$ and it is a practical and efficient method that uses current CLSI methodology to detect plasmid-mediated AmpC $\beta$ lactamase (PABL) in organisms that usually do not harbor genes for these enzymes. It is unclear why the disk test missed one of the AmpC-producing Proteus mirabilis isolates, although it may be due to swarming phenomena, which is often seen with this organism on agar media.

In a study performed by Hemalatha and his colleagues in 2007 (19), a total number of 76 clinical isolates $(67$ E. coli and 9 Klebsiella pneumoniae) were screened for AmpC production by disk diffusion method using

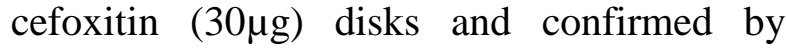
inhibitor based test using boronic acid as inhibitor. A total of 36 of 76 isolates (47.3\%) screened were harboured AmpC enzymes, of which a majority $31(86.1 \%)$ co-produced ESBL enzymes. Pure AmpC production was seen in 7 out of $76(9.2 \%)$ of isolates only. The inhibitor based test was useful in identifying cefoxitin susceptible AmpC producers and could also effectively differentiate ESBL from AmpC producing isolates.

In our study we found that the sensitivity of combination-disk test with boronic acid was $58.6 \%$. Hemalatha and his colleagues 2007 (19) found that the sensitivity was $47.3 \%$. The detection failure could be due to very high levels of AmpC expression and detection could possibly be improved by increasing the concentration of the enzyme inhibitor (boronic acid).

In our study, cephamycin-Hodge test showed higher sensitivity (75.9\%), specificity $(100.0 \%)$ and efficacy $(86.0 \%)$ than TrisEDTA disk test $(37.9 \%, 100.0 \%$ and $64.0 \%$ respectively) and combination-disk test with boronic acid $(58.6 \%, 100.0 \%$ and $76.0 \%$ respectively).

In agreement with our results, Shanthi et al., 2012, (20) screened 77 isolates, K. pneumonia $(\mathrm{n}=52)$ and E. coli $(\mathrm{n}=25)$ for AmpC production by disk diffusion and MIC determination using cefoxitin. These isolates were then subjected to cefoxitin Hodge test and boronic acid inhibitor method. The presence of AmpC genes was confirmed by multiplex PCR. The Boronic acid Inhibition (BAI) test was positive in 55 isolates which included $19 \mathrm{E}$. coli and $36 \mathrm{~K}$. pneumoniae. Hodge test using cefoxitin was positive in 40 isolates which included 20 of each E. coli and $K$. pneumoniae. Multiplex PCR detected plasmid Amp C in 23 isolates, of which 12 were $K$. pneumoniae and 11 were E. coli. Considering PCR as the gold standard, they compared the sensitivity and specificity of the 
phenotypic tests employed. The Hodge test fared better in terms of sensitivity and specificity when compared with the inhibitorbased test $(78.2 \%$ and $59.2 \%$ vs $65.2 \%$ and $25.9 \%$ ). They concluded that PCR and isoelectric focusing remain the gold standard for detection of AmpC. The phenotypic tests for Amp C detection have low sensitivity and specificity as multiple Amp C types coexist in many isolates and presence of $\beta$-lactamases and/or porin defects render the phenotypic tests unreliable since many of these mechanisms mask each other.

In contrast to our results, Lee et al., 2009 (21) studied 276 clinical isolates of $E$. coli $(\mathrm{N}=97)$, $K$. pneumoniae $(\mathrm{N}=136)$, and $P$. mirabilis (N=43). Multiplex PCR was performed to detect the PABL genes. Further, 3 phenotypic detection methods cephamycin-Hodge test, Tris-EDTA (TE) disk test, and combinationdisk test with 3-aminophenylboronic acid (BA) were performed using cefoxitin and cefotetan disks. They found that PABL genes were detected by multiplex PCR in 122/276 isolates, including 14/97 E. coli, 105/136 K. pneumoniae, and 3/43 P. mirabilis isolates. A total of 93 PABL-producing strains were positive with the cephamycin-Hodge test, but 29 PABL-producing strains were not detected by this method. By TE -disk test, 98 strains were determined as PABL-producers, while 24 strains were false-negatives. Combinationdisk test with BA detected 120 strains of the 122 PABL-producers, but 12 PABL nonproducers were also tested positive by this method. The combination-disk test with BA showed sensitivity $(98.4 \%)$, specificity $(92.2 \%)$, and efficiency $(96.3 \%)$ and the cephamycin-Hodge showed $(76.2 \%, 96.1 \%$, and $88.6 \%$, respectively) and the TE-disk test (80.3\%, 91.6\%, and $87.9 \%$, respectively). They concluded that the combination-disk test with BA is a simple, efficient, and interpretable test that can be applicable in clinical laboratories for detection of PABLs in clinical isolates of E. coli, K. pneumoniae, and $P$. mirabilis.

\section{Acknowledgment}

The authors would like to thank NAMRU- 3 for providing the E. coli ATCC 25922 strain.

\section{References}

1. Jameel N., Ejaz H., Zafar A and Amin H. 2014. Multidrug resistant AmpC $\beta$-lactamases producing Escherichia coli isolated from a paediatric hospital. Pak J Med Sci., 30(1):181184.

2. Song W., Kim J., Jeong H.S., Seo H.Y., Shin H.J., Jang J.S., Uh Y., Lee M., and Lee K. 2011.Chromosome encoded AmpC and CTX$\mathrm{M}$ extended-spectrum $\beta$-lactamases in clinical isolates of Proteus mirabilis from Korea. Antimicrob. Agents Chemother. 55(4): 14141419.

3. Jawetz, Melnick., and Adelberg's. 2007.Enteric gram negative rods (Enterobacteriaceae) in Jawetz, Melnick, and Adelberg's Medical Microbiology, 24th edition, chapter 16, The McGraw-Hill Companies.

4. French G. 2010. The continuing crisis in antibiotic resistance. Intern. J. Antimicrob., Agents. 36(3): 3-7.

5. Moland E. S., Kim S., Hong S. G., and Thomson K. S. 2008. Newer $\beta$-lactamases: clinical and laboratory implications, part II. Clinical Microbiology Newsletter., 30 (11): 79-85.

6. Doi $\mathrm{Y}$ and Paterson D. 2007.Detection of plasmid-mediated class $\mathrm{C} \beta$-lactamases. International Journal of Infectious Diseases. 11(3): 191-197.

7. Yong D., Park R., Yum J., Lee K., Choi E, and Chong Y. 2002. Further modification of the Hodge test to screen AmpC $\beta$-lactamase (CMY-1)-producing strains of Escherichia coli and Klebsiella pneumoniae. Journal of Microbiological Method., 51(3): 407-410.

8. Black J., Moland E andThomson K. 2005.AmpCdisktest for detection of plasmidmediated AmpC $\beta$-lactamases in Enterobacteriaceae lacking chromosomal 
AmpC $\beta$-lactamases. Journal of Clinical Microbiology., 43(7): 3110-3113.

9. Tan T., Young L., He J., Hsien T and Yang Li. 2009.Evaluationof screening methods to detect plasmid-mediated AmpC in Escherichia coli, Klebsiellapneumoniae, and Proteus mirabilis., Antimicrobial Agents and Chemotherapy., 53(1): 146-149.

10. Brenwald NP., Jevons G., Andrews., Ang L and Fraise AP. 2005.Disc methods for detecting AmpC $\beta$-lactamase-producing clinical isolates of Escherichia coli and Klebsiella pneumoniae. Journal of Antimicrobial Chemotherapy., 56(3): 600601.

11. Livermore D., Warner $M$, and Mushtaq S. 2007.Evaluation of the chromogenic Cica- $\beta$ Test for detecting extended-spectrum,AmpC and metallo- $\beta$-lactamases. Journal of Antimicrobial Chemotherapy., 60(6): 13751379.

12. Yagi T., Wachino J., KurokawaH et al., 2005.Practical methods using boronic acid compounds for identification of class $\mathrm{C} \beta$ lactamase-producing Klebsiella pneumoniae and Escherichia coli. Journal of Clinical Microbiology., 43(6): 2551-2558.

13. Nasim K., Elsayed S.J.D., Pitout D., Conly J., Church D.L and Gregson D.B. 2004. New Method for Laboratory Detection of AmpC $\beta$ Lactamases in Escherichia coli and Klebsiella pneumoniae. J Clin Microbiol., 42(10): 47994802.

14. Singhal S., Mathur T., Khan S., Upadhyay D.J., Chugh S., Gaind R and Rattan A.2005. Evaluation of methods for AmpC $\beta$ lactamase in gram negative clinical isolates from tertiary care hospitals. Indian $\mathrm{J}$ Med Microbiol., 23(2):120-4.

15. Song W., Bae K., Lee Y.N., Lee C.H., Lee S.H and Jeong S.H. 2007. Detection of
Extended-Spectrum B-Lactamases by Using Boronic Acid as an AmpC B-Lactamase Inhibitor in Clinical Isolates of Klebsiella spp. and Escherichia coli. Journal of Clinical Microbiology., 45(4135): 1180-1184.

16. Pérez-Pérez F.J. and Hanson N.D. 2002. Detection of Plasmid-Mediated AmpC BLactamase Genes in Clinical Isolates by Using Multiplex PCR Journal of Clinical Microbiology., 40(6):2153-2162.

17. Alper E., ÖzenKoseogiu E., and Gülsen H. 2011. Erythromycin and penicillin resistance mechanisms among viridans group streptococci isolated from blood cultures of adult patients with underlying diseases. New Microbiologica., 34, 187-193.

18. Coudron P. E. 2005.Inhibitor-Based Methods for Detection of Plasmid-Mediated AmpC $-\beta$ Lactamases in Klebsiella spp., Escherichia coli, and Proteus mirabilis. J. Clin. Microbiol., 43(8): 4163-4167.

19. Hemalatha V., Padma M., Sekar U., Vinodh T.M., Arunkumar A.S. 2007. Detection of Amp C beta lactamases production in Escherichia coli \& Klebsiella by an inhibitor based method. Indian J Med Res., 126(3):220223.

20. Shanthi M., Sekar U., Arunagiri Kand Sekar B. 2012.Detection of Amp C genes encoding for beta-lactamases in Escherichia coli and Klebsiella pneumoniae. Indian J Med Microbiol., 30(3):290-295.

21. Lee W., Jung B., Hong G.S., Song W., Jeong H.S., Lee K., and Kwak H. 2009.Comparison of 3 phenotypic-detection methods for identifying plasmid-mediated AmpC $\beta$ lactamase producing Escherichia coli, Klebsiella pneumoniae, and Proteus mirabilis strains. Korean J Lab Med., 29:448-54.

\section{How to cite this article:}

Rania A. Amar and Karim A. Montasser. 2017. Evaluation of Different Phenotypic Methods Versus Polymerase Chain Reaction for Detection of Plasmid Mediated AmpC $\beta$-LactamaseProducing Strains of Proteus mirabilis. Int.J.Curr.Microbiol.App.Sci. 6(11): 4201-4210. doi: https://doi.org/10.20546/ijcmas.2017.611.492 